\title{
Investigation of chromosome $Y$ loss in men with schizophrenia
}

This article was published in the following Dove Press journal:

Neuropsychiatric Disease and Treatment

\section{Takashi Hirata \\ Akitoyo Hishimoto \\ Ikuo Otsuka \\ Satoshi Okazaki \\ Shuken Boku \\ Atsushi Kimura \\ Tadasu Horai \\ Ichiro Sora}

Department of Psychiatry, Kobe University Graduate School of Medicine, Kusunoki-cho, Chuo-ku, Kobe, Japan
Correspondence: Akitoyo Hishimoto Department of Psychiatry, Kobe University Graduate School of Medicine,

7-5-I, Kusunoki-cho, Chuo-ku,

Kobe 650-0017, Japan

Email hishipon@med.kobe-u.ac.jp
Background: Life expectancy is 10-20 years lower in patients with schizophrenia than in the general population. In addition, men with schizophrenia have an earlier age at onset, more pronounced deficit symptoms, poorer course, and poorer response to antipsychotic medications than women. Recent studies have indicated that loss of chromosome Y (LOY) in peripheral blood is associated with an increased risk of all-cause mortality. In order to elucidate the pathophysiology of male-specific features, we investigated the association between LOY and schizophrenia.

Materials and methods: The present study included 360 Japanese men (146 patients with schizophrenia vs 214 controls). The relative amount of $\mathrm{Y}$ chromosome was defined as the ratio of chromosome $\mathrm{Y}$ to chromosome $\mathrm{X}$ (Y/X ratio) based on the fluorescent signal of co-amplified short sequences from the $\mathrm{Y}-\mathrm{X}$ homologous amelogenin genes (AMELY and AMELX).

Results: There was no significant difference in the frequency of LOY between the schizophrenia and control groups. However, longer duration of illness was associated with LOY after controlling for age and smoking status in the schizophrenia group $(P=0.007$, OR $=1.11[95 \%$ $\mathrm{CI}=1.03-1.19])$.

Conclusion: According to our results, schizophrenia may not have a remarkable effect on blood LOY; however, LOY may be associated with disease course in patients with schizophrenia.

Keywords: loss of chromosome Y, schizophrenia, disease duration

\section{Introduction}

Schizophrenia is a severe psychiatric disorder that exerts a profound impact on the individual and society, primarily due to chronic negative symptoms such as impaired motivation, reductions in spontaneous speech, and cognitive impairment. Furthermore, it is well-known that mortality rates for schizophrenia are extremely high. Indeed, several studies have suggested that schizophrenia is associated with an increased risk of mortality, reducing the life expectancy of affected individuals by 10-20 years relative to that in the general population. ${ }^{1,2}$ For several decades, schizophrenia was generally believed to be associated with a uniform lifetime morbid risk of $1 \%,{ }^{3}$ although these effects may be influenced by smoking and other lifestyle factors.

Numerous family studies, twin and adoption studies, candidate gene analyses, and genome-wide association studies (GWASs) have suggested that genetic factors contribute to the etiology of schizophrenia. ${ }^{4}$ In addition, several features of schizophrenia are thought to be sex-specific, as the incidence of the disorder is 1.4 times higher among men than among women. Relative to women, men with schizophrenia exhibit an earlier age at onset, poorer premorbid history, a higher frequency of prenatal and perinatal complications, more pronounced deficits, poorer course, and poorer response to typical antipsychotic medications. ${ }^{5}$ Despite these findings, it remains unclear how sex differences influence the pathophysiology of schizophrenia. 
Male-specific genetic risk factors such as loss of chromosome Y (LOY) in blood cells may help to elucidate the role of sex differences in schizophrenia. LOY is primarily discussed as a mosaic event, in which a mixture of cells with and without the $\mathrm{Y}$ chromosome can be observed in the peripheral blood of normally aging men. ${ }^{6}$ At the cellular level, LOY is a binary phenomenon: that is, a cell either has the Y chromosome or does not. Several studies have reported LOY in men of advanced age, suggesting that LOY is associated with aging. ${ }^{7-11}$ Other studies have demonstrated that smoking is a major risk factor for LOY in the blood. ${ }^{11,12}$ Further, the median survival time after sampling of men with LOY is 5.5 years shorter than that observed in controls. ${ }^{13}$ Several research groups have also observed increased LOY in various diseases, such as hematological malignancies, ${ }^{14}$ non-hematological tumors, ${ }^{15}$ Alzheimer's disease, ${ }^{9}$ and autoimmune thyroiditis. ${ }^{16}$ Fontenelle et al further reported an association between LOY and Asperger's syndrome, ${ }^{17}$ and our recent study demonstrated that increased LOY can be observed in those who have completed suicide. ${ }^{18}$ However, no previous study has examined the association between LOY and schizophrenia. In the present study, we conducted the first investigation of LOY in patients with schizophrenia.

\section{Materials and methods}

\section{Participants}

The present study was approved by the Ethical Committee for Genetic Studies of Kobe University Graduate School of Medicine, and our study design and all related procedures were performed in accordance with the Declaration of Helsinki. All participants provided written informed consent, were of Japanese descent, and were recruited from among the population of Kobe, Japan.
This study cohort consisted of 146 men with schizophrenia (median age: 60.0 years, interquartile range [IQR 49.0-68.0] years) and 214 age-matched controls (214 men; median age: 58.5 years, [IQR 43.0-68.0] years). The demographic and clinical characteristics of the participants are presented in Table 1.

All patients with schizophrenia were diagnosed by at least two psychiatrists in accordance with criteria outlined in the Diagnostic and Statistical Manual of Mental Disorders, fourth edition (DSM-IV) or DSM-5 based on unstructured interviews and reviews of their medical records. The control group included healthy volunteers. None of the control participants had any present, past, or family (first-degree relatives) history of psychiatric disorders or substance abuse, excluding nicotine dependence. All control participants were interviewed and screened for psychiatric disorders based on an unstructured interview performed by a psychiatrist. We obtained information regarding smoking status for each patient with schizophrenia, although such information was not available for control participants.

\section{Determination of LOY in peripheral blood}

Peripheral blood samples were stored at $-80^{\circ} \mathrm{C}$ prior to analysis. DNA was extracted using the QIAamp DNA Blood Midi Kit (Qiagen NV, Venlo, the Netherlands), in accordance with the manufacturer's instructions. Extracted DNA was quantified and underwent quality control using a NanoDrop spectrophotometer (Thermo Fisher Scientific, Waltham, MA, USA). LOY was determined using quantitative fluorescence polymerase chain reaction as previously described. . $^{15,19,20}$

The relative amount of $Y$ chromosome was defined as the ratio of chromosome $\mathrm{Y}$ to chromosome $\mathrm{X}$ ( $\mathrm{Y} / \mathrm{X}$ ratio) based on the fluorescent signal of co-amplified short sequences

Table I Demographic and clinical characteristics of participants for loss of chromosome $Y$ study

\begin{tabular}{|c|c|c|c|}
\hline & $\begin{array}{l}\text { Control } \\
(n=214)\end{array}$ & $\begin{array}{l}\text { Schizophrenia } \\
(n=\mid 46)\end{array}$ & $P$-value \\
\hline Age (years) (median [IQR]) & $58.5[43.0-68.0]$ & $60.0[49.0-68.0]$ & $0.125^{\mathrm{a}}$ \\
\hline Smoking (never/former/current) ${ }^{\mathrm{b}}$ & - & $42 / 43 / 61$ & - \\
\hline Age of onset (years) (median [IQR]) & - & $25.0[20.0-32.0]$ & - \\
\hline Duration of illness (years) (median [IQR]) & - & $33.0[18.0-42.0]$ & - \\
\hline Antipsychotic dose (mg/day) (median [IQR]) ${ }^{c}$ & - & $675.0[375.0-1,250.0]$ & - \\
\hline $\mathrm{GAF}$ score $($ mean $\pm \mathrm{SD})$ & - & $38.0 \pm 7.7$ & - \\
\hline BPRS score (median [IQR]) & - & $42.0[39.0-53.0]$ & - \\
\hline Presence of LOY (with LOY/without LOY) & $18 / 196$ & $13 / 133$ & $0.85 I^{d}$ \\
\hline
\end{tabular}

Notes: Accurate information regarding age of onset and duration of illness was available from the clinical records of 145 of 146 patients with schizophrenia. Accurate information regarding GAF and BPRS scores were available from the clinical records of 69 of 146 patients with schizophrenia. ${ }^{a}$-value between schizophrenia and control groups calculated using Mann-Whitney U-test. 'For smoking status, "never" refers to an individual who has never been a cigarette or cigar smoker, while "former" indicates an individual who had smoked in his lifetime but who was not a smoker at the time of blood sampling, and "current" refers to an individual who was a smoker at the time of blood sampling. 'Antipsychotic dose was calculated based on chlorpromazine equivalents. dP-value between individuals with and without LOY calculated using Fisher's exact test. Abbreviations: IQR, interquartile range; LOY, loss of chromosome Y; GAF, Global Assessment of Functioning; BPRS, Brief Psychiatric Rating Scale. 
from the $\mathrm{Y}-\mathrm{X}$ homologous amelogenin genes ( $A M E L Y$ and $A M E L X)$. The ratio was discriminated based on a 6-base pair (bp) deletion in intron 1 of $A M E L X$ (106-bp fragment), which is not found in AMELY (112-bp fragment). Deletions or duplications in the $A M E L Y$ region were excluded using a co-amplified homologous sequence primer set for MYPT2 on chromosome 1 (176-bp fragment) and MYPT2 on chromosome Y (two copies of 181-bp fragments). In addition, using another co-amplified homologous sequence set for $T A F 9 B$ on chromosome 3 (140-bp fragment) and the X chromosome (144-bp fragment), we verified that there were no false positive cases of $\mathrm{X}$ chromosome loss or gain in the present study. An $A M E L Y / X$ ratio of 1 indicates a normal result for male DNA samples due to the presence of one $\mathrm{X}$ and one $\mathrm{Y}$ chromosome. The normal MYPT2/MYPT2Y ratio is approximately one due to the presence of two copies of the gene on the $\mathrm{Y}$ chromosome and one copy on each copy of chromosome 1. The normal TAF9B-X/TAF9B-chr 3 ratio is 0.5 due to the presence of two copies of the gene on chromosome 3 and one copy on the $\mathrm{X}$ chromosome in male individuals.

The polymerase chain reaction (PCR) reaction mixture contained 2.5 $\mu \mathrm{L}$ AmpliTaq Gold 360 Master Mix (Applied Biosystems, Thermo Fisher Scientific), 20 ng genomic DNA, and 3 pmol of each primer, resulting in a total volume of $10 \mu \mathrm{L}$. PCR was performed under the following conditions: initial denaturation at $95^{\circ} \mathrm{C}$ for 5 minutes, 28 cycles denaturation cycles at $95^{\circ} \mathrm{C}$ for 1 minute each, annealing at $58^{\circ} \mathrm{C}$ for 1 minute, elongation at $72^{\circ} \mathrm{C}$ for 1.5 minute, and final elongation at $72^{\circ} \mathrm{C}$ for 30 minutes. PCR products were run on an Applied Biosystems 3,130 XL Genetic Analyzer (Applied Biosystems, Thermo Fisher Scientific) with GeneScan 600 LIZ (Applied Biosystems, Thermo Fisher Scientific) as an internal standard and were analyzed using GeneMapper version 4.1 (Applied Biosystems, Thermo Fisher Scientific). Because co-amplified fragments from the $\mathrm{X}$ and $\mathrm{Y}$ chromosomes exhibit a 6-bp difference (106 bp vs $112 \mathrm{bp}$, respectively), they are readily separated and quantified via capillary electrophoresis. Laboratory personnel were blinded regarding case-control status, and the sample order was randomized in each batch.

\section{Statistical analysis}

Statistical analysis was performed using $\mathrm{R}$ version 3.4.1 (The R Foundation for Statistical Computing, Vienna, Austria) equipped with EZR version 1.36. ${ }^{21}$ Differences between groups were analyzed using Mann-Whitney $U$-tests, Fisher's exact tests, Cochran-Armitage trend tests, and logistic regression analysis, as appropriate. Relationships between continuous variables were analyzed using Spearman's rank correlation coefficient. We evaluated the presence or absence of LOY, coding Y/X ratios $<0.9$ as LOY and Y/X ratios $\geq 0.9$ as normal. This threshold was based on previous findings, which revealed that detection of LOY from single nucleotide polymorphism (SNP)-array data is robust and reproducible when LOY occurs in $\geq 10 \%$ of the nucleated cells in a blood sample. ${ }^{6,12,13}$ Dummy variables were used as necessary (phenotype, control $=0$, schizophrenia $=1$; smoking, no $=0$, past $=1$, yes $=2$ ). Statistical significance was defined as two-tailed $P$-values $<0.05$.

\section{Results LOY in peripheral blood}

Spearman's correlation coefficients revealed negative correlations between age and $\mathrm{Y} / \mathrm{X}$ ratio in both the schizophrenia $(r=-0.329, P<0.001)$ and control $(r=-0.294, P<0.001)$ groups (Figure 1), in accordance with the findings of previous studies. ${ }^{7-11}$ Thirteen of $146(8.9 \%)$ patients and 18 of 214 (8.4\%) controls exhibited LOY (Y/X ratio $<0.9)$. As such, there was no significant difference in the frequency of LOY between the schizophrenia and control groups ( $P=0.851$ ) (Table 1). Logistic regression analysis revealed no significant effect of age on phenotype $(P=0.982)$ (Table 2$)$.

Next, we investigated the effect of schizophrenia duration on LOY. We observed a positive correlation between the duration of illness and age in the schizophrenia group $(r=0.776, P<0.001)$ (Figure S1). To adjust for the effects of age bias in subsequent analysis, we utilized data from patients with schizophrenia over the age of 53 - the lower limit for LOY observed in the present study. In this subgroup, the duration of illness was significantly longer in patients with LOY than in those without LOY $(P=0.0016)$, and there were no significant differences in age between patients with LOY and those without LOY $(P=0.077)$ (Table S1 and Figure 2). Moreover, we observed no effects of smoking status or antipsychotic dose. To rule out potential confounders, we performed a logistic regression analysis, in which LOY was the response variable, while duration of illness, smoking status, and antipsychotic dose were the explanatory variables. The duration of illness was robustly associated with LOY ( $P=0.007, \mathrm{OR}=1.11$ [95\% CI $=1.03-1.19])$ (Table 3$)$. However, no significant effect of age was observed $(P=0.109)$ (Table S2).

\section{Discussion}

To our knowledge, the present study is the first investigation of LOY among patients with schizophrenia. Because 

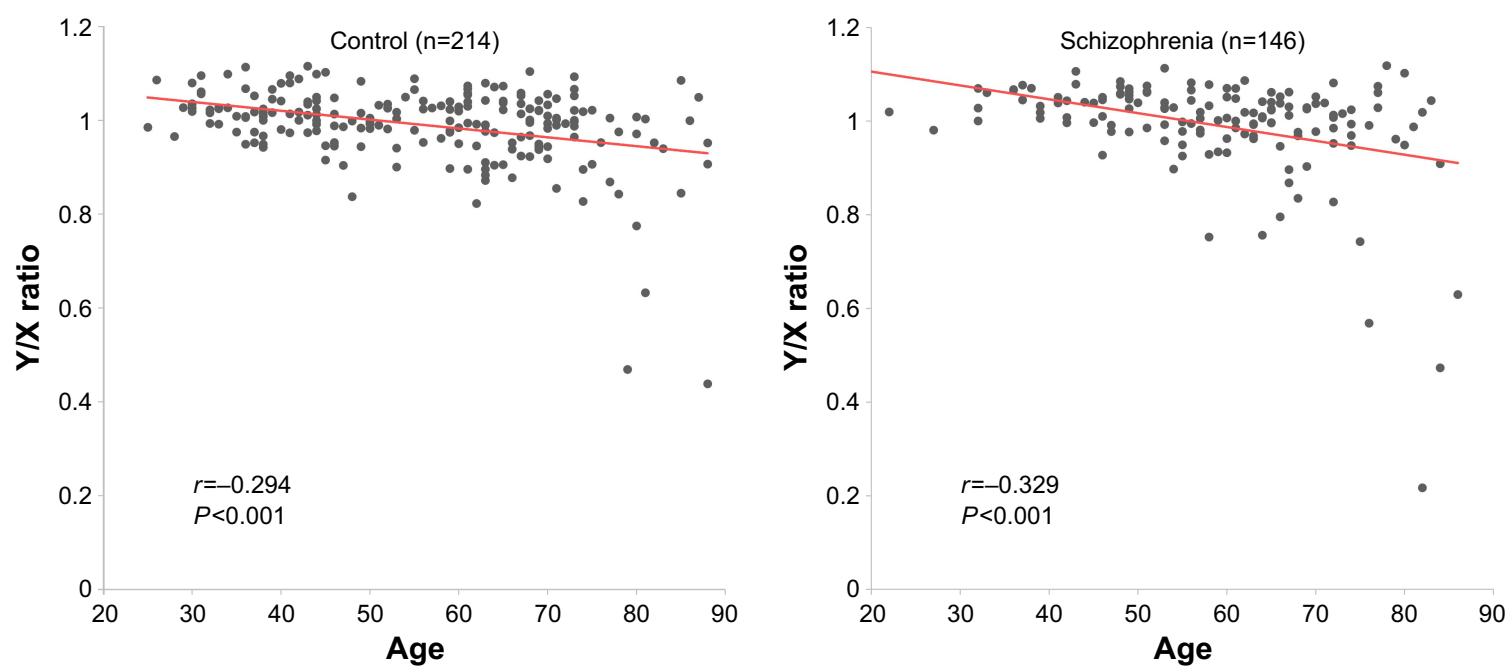

Figure I Relationship between age and Y/X ratio in blood samples from patients with schizophrenia and controls. All $P$-values and $r$ values were calculated using Spearman's rho test.

the association between age and LOY has been well documented, we focus on age-adjusted comparisons between the groups. In accordance with previous findings, our analyses revealed an association between LOY and age in both the schizophrenia and control groups. ${ }^{7-11}$ However, there was no significant difference in LOY between the two groups. In contrast, we observed that longer duration of schizophrenia was associated with LOY regardless of smoking status or daily antipsychotic dose.

Our results suggested that LOY was correlated with the duration of schizophrenia, but not with disease status. The association between age and LOY has been well documented. In addition, several research groups have also observed increased LOY in patients with aging-associated diseases, such as myelodysplastic syndrome ${ }^{22}$ and Alzheimer's disease. ${ }^{9}$ Taken together, these findings indicate that increases in LOY may also occur over a prolonged period in patients with schizophrenia.

Several studies have demonstrated that smoking is a major risk factor for LOY, and that the mutagenic effects are transient and dose-dependent. ${ }^{11,12}$ As smoking data were

Table 2 Logistic regression analysis of LOY in peripheral blood samples from patients with schizophrenia and controls

\begin{tabular}{lllll}
\hline Explanatory variable & \multicolumn{4}{l}{$\begin{array}{l}\text { Response variable: LOY } \\
\text { (Y/X ratio }<\mathbf{0 . 9})\end{array}$} \\
\cline { 2 - 5 } & $\mathbf{B}^{\mathbf{a}}$ & $\mathbf{P}$-value & OR & $\mathbf{9 5 \%} \mathbf{~ C l}$ \\
\hline Phenotype (CON vs SCZ) & 0.01 & 0.982 & 1.01 & $0.46-2.22$ \\
Age & 0.09 & $<\mathbf{0 . 0 0 1}$ & 1.09 & $1.05-1.13$ \\
\hline
\end{tabular}

Notes: For logistic regression analyses, LOY was the response variable, while phenotype and age were the explanatory variables. Boldface type indicates statistical significance $(P<0.05)$. ${ }^{a} B$ represents the unstandardized partial regression coefficient. Abbreviations: LOY, loss of chromosome Y; SCZ, schizophrenia; CON, control. unavailable for control participants, we referred to public epidemiological data for smoking rates in Japan..$^{23}$ Our analysis revealed that patients with schizophrenia exhibited a higher average smoking rate than that observed in the

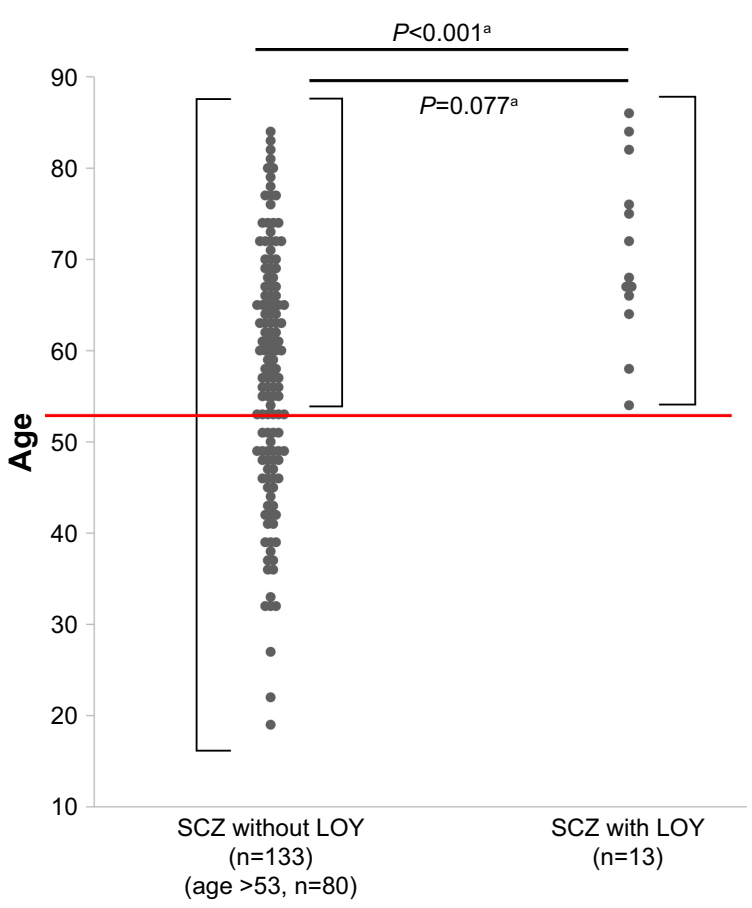

Figure 2 Dot plot of age for men with and without LOY in schizophrenia. Notes: The red line represents 53 years of age. Significant differences in age were observed between patients with schizophrenia exhibiting LOY and those without LOY (median age: 68.0 years, IQR [66.0-76.0] years vs median age: 58.0 years, IQR [48.0-67.0] years) $(P<0.00 \mathrm{I})$. When the analysis was limited to patients over age 53 , there was no significant difference between patients with and without LOY (median age: 68.0 years, IQR [66.0-76.0] years vs median age: 65.0 years, IQR [60.0-72.0] years) $(P=0.077)$. a $P$-values between patients with and without LOY were calculated using Mann-Whitney U-tests.

Abbreviations: LOY, loss of chromosome Y; SCZ, schizophrenia; IQR, interquartile range. 
Table 3 Logistic regression analysis of LOY in peripheral blood samples of patients with schizophrenia

\begin{tabular}{lllll}
\hline Explanatory variable & \multicolumn{4}{l}{$\begin{array}{l}\text { Response variable: LOY } \\
(\mathbf{Y} / \mathbf{X} \text { ratio }<\mathbf{0 . 9})\end{array}$} \\
\cline { 2 - 5 } & $\mathbf{B}^{\mathbf{a}}$ & $\mathbf{P}$-value & $\mathbf{O R}$ & $\mathbf{9 5 \%} \mathbf{~ C l}$ \\
\hline Duration of illness & 0.100 & $\mathbf{0 . 0 0 7}$ & $\mathrm{I} . \mathrm{II}$ & $1.03-1.19$ \\
Smoking status & -0.230 & 0.560 & 0.79 & $0.36-1.74$ \\
Antipsychotic dose & $-0.000 \mathrm{I}$ & 0.894 & $\mathrm{I} .00$ & $0.99-1.00$ \\
\hline
\end{tabular}

Notes: For logistic regression analyses, LOY was the response variable, while duration of illness, smoking, and antipsychotic dose were the explanatory variables. Boldface type indicates statistical significance $(P<0.05)$. ${ }^{\mathrm{B}} \mathrm{B}$ represents the unstandardized partial regression coefficient.

Abbreviation: LOY, loss of chromosome $Y$.

general population ( $41.7 \%$ vs $34.1 \%$; Table S3), in accordance with the findings of previous studies. ${ }^{24,25}$ However, we observed no significant difference in LOY between the schizophrenia and control groups, suggesting that differences in smoking rate do not influence the effect of smoking on LOY.

Recent European GWASs found that LOY was related to cell cycle regulation and genome instability. ${ }^{10,11} \mathrm{We}$ hypothesized that disturbed cell cycle regulation and genomic instability play important roles in the pathophysiology of schizophrenia. Indeed, several studies have demonstrated that schizophrenia involves the aberrant regulation of cell cycle-related genes in the brain and peripheral blood, along with chromosomal aneuploidy in the brain. ${ }^{26-31}$ Furthermore, a recent high-resolution genome-wide copy number variation (CNV) analysis using data derived from the Japanese population suggests that genomic instability is associated with the pathophysiology of schizophrenia, mainly due to genomic replication errors. ${ }^{32}$ However, no studies to date have revealed mechanistic links between genomic instability and schizophrenia. Further research is required to elucidate the mechanisms underlying the pathophysiology of schizophrenia.

There are several limitations in the present study. First, clinical information regarding smoking status, a factor known to affect LOY, ${ }^{11,12}$ was not available for our control participants. Although we investigated the influence of smoking on LOY using publicly available epidemiological data, it is unclear whether this influence was similar in our control participants. Future studies should include detailed information regarding smoking status (ie, cigarettes per day, duration of smoking) for both the schizophrenia and control groups. Second, we adjusted age to compare the duration of illness between participants with and without LOY in the schizophrenia group. However, precise elimination of the age effect requires an age-static cohort, in which all participants are sampled at the same age. Third, our calculations of antipsychotic dose may have been inaccurate, as these values reflected those at the time of blood sampling, and we did not integrate medication history. Finally, the sample size of our study may have been insufficient for detecting associations between schizophrenia and LOY. Larger sample sizes are required in future studies.

\section{Conclusion}

This study indicated that schizophrenia may not have a remarkable effect on blood LOY; however, LOY might be involved in the disease course of schizophrenia. Our findings shed light on further investigation of LOY toward an improved understanding of the sex-specific features of schizophrenia.

\section{Acknowledgments}

This work was supported in part by the Japan Society for the Promotion of Science (JSPS) KAKENHI Grant Numbers JP15K19727 and JP16K19765. We thank Yasuko Nagashima for providing technical assistance.

\section{Disclosure}

The authors report no conflict of interest in this work.

\section{References}

1. Chesney E, Goodwin GM, Fazel S. Risks of all-cause and suicide mortality in mental disorders: a meta-review. World Psychiatry. 2014; 13(2):153-160.

2. Saha S, Chant D, McGrath J. A systematic review of mortality in schizophrenia: is the differential mortality gap worsening over time? Arch Gen Psychiatry. 2007;64(10):1123-1131.

3. Mueser KT, McGurk SR. Schizophrenia. Lancet. 2004;363(9426): 2063-2072.

4. Sullivan PF, Kendler KS, Neale MC. Schizophrenia as a complex trait: evidence from a meta-analysis of twin studies. Arch Gen Psychiatry. 2003;60(12):1187-1192.

5. Abel KM, Drake R, Goldstein JM. Sex differences in schizophrenia. Int Rev Psychiatry. 2010;22(5):417-428.

6. Forsberg LA. Loss of chromosome Y (LOY) in blood cells is associated with increased risk for disease and mortality in aging men. Hum Genet. 2017;136(5):657-663.

7. Wiktor AE, van Dyke DL, Hodnefield JM, Eckel-Passow J, Hanson CA. The significance of isolated $\mathrm{Y}$ chromosome loss in bone marrow metaphase cells from males over age 50 years. Leuk Res. 2011;35(10): 1297-1300.

8. Jacobs PA, Maloney V, Cooke R, Crolla JA, Ashworth A, Swerdlow AJ. Male breast cancer, age and sex chromosome aneuploidy. $B r J$ Cancer. 2013;108(4):959-963.

9. Dumanski JP, Lambert JC, Rasi C, et al. Mosaic loss of chromosome Y in blood is associated with Alzheimer disease. Am J Hum Genet. 2016; 98(6):1208-1219.

10. Wright DJ, Day FR, Kerrison ND, et al. Genetic variants associated with mosaic Y chromosome loss highlight cell cycle genes and overlap with cancer susceptibility. Nat Genet. 2017;49(5):674-679.

11. Zhou W, Machiela MJ, Freedman ND, et al. Mosaic loss of chromosome $\mathrm{Y}$ is associated with common variation near TCL1A. Nat Genet. 2016;48(5):563-568. 
12. Dumanski JP, Rasi C, Lönn M, et al. Mutagenesis. Smoking is associated with mosaic loss of chromosome Y. Science. 2015;347(6217): 81-83.

13. Forsberg LA, Rasi C, Malmqvist N, et al. Mosaic loss of chromosome Y in peripheral blood is associated with shorter survival and higher risk of cancer. Nat Genet. 2014;46(6):624-628.

14. Pierre RV, Hoagland HC. Age-associated aneuploidy: loss of Y chromosome from human bone marrow cells with aging. Cancer. 1972; 30(4):889-894.

15. Noveski P, Madjunkova S, Sukarova Stefanovska E, et al. Loss of Y chromosome in peripheral blood of colorectal and prostate cancer patients. PLoS One. 2016;11(1):e0146264.

16. Persani L, Bonomi M, Lleo A, et al. Increased loss of the Y chromosome in peripheral blood cells in male patients with autoimmune thyroiditis. J Autoimmun. 2012;38(2-3):J193-J196.

17. Fontenelle LF, Mendlowicz MV, Bezerra de Menezes G, dos Santos Martins RR, Versiani M. Asperger syndrome, obsessive-compulsive disorder, and major depression in a patient with 45, X/46, XY mosaicism. Psychopathology. 2004;37(3):105-109.

18. Kimura A, Hishimoto A, Otsuka I, et al. Loss of chromosome Y in blood, but not in brain, of suicide completers. PLoS One. 2018;13(1): e0190667.

19. Sullivan KM, Mannucci A, Kimpton CP, Gill P. A rapid and quantitative DNA sex test: fluorescence-based PCR analysis of X-Y homologous gene amelogenin. Biotechniques. 1993;15(4):636-638, 640-641.

20. Plaseska Karanfilska D, Noveski P, Plaseski T. Detection of the most common genetic causes of male infertility by quantitative fluorescent (QF)-PCR analysis. In: Plaseska-Karanfilska D, editor. Human Genetic Diseases. InTech, 2011. ISBN: 978-953-307-936-3. Available from: https://www.intechopen.com/books/human-genetic-diseases/ detection-of-the-most-common-genetic-causes-of-male-infertility-byquantitative-fluorescent-qf-pcr-a. Accessed August 08, 2018.

21. Kanda Y. Investigation of the freely available easy-to-use software 'EZR' for medical statistics. Bone Marrow Transplant. 2013;48(3): $452-458$.
22. Ganster C, Kämpfe D, Jung K, et al. New data shed light on Y-loss-related pathogenesis in myelodysplastic syndromes. Genes Chromosomes Cancer. 2015;54(12):717-724.

23. NIBIOHN [webpage on the Internet]. The National Health and Nutrition Survey Japan; 2012. Available from: http://www.nibiohn.go.jp/eiken/ english/research/project_nhns.html. Accessed July 27, 2018.

24. de Leon J, Dadvand M, Canuso C, White AO, Stanilla JK, Simpson GM. Schizophrenia and smoking: an epidemiological survey in a state hospital. Am J Psychiatry. 1995;152(3):453-455.

25. Ziedonis D, Williams JM, Smelson D. Serious mental illness and tobacco addiction: a model program to address this common but neglected issue. Am J Med Sci. 2003;326(4):223-230.

26. Katsel P, Davis KL, Li C, et al. Abnormal indices of cell cycle activity in schizophrenia and their potential association with oligodendrocytes. Neuropsychopharmacology. 2008;33(12):2993-3009.

27. Benes FM, Lim B, Subburaju S. Site-specific regulation of cell cycle and DNA repair in post-mitotic GABA cells in schizophrenic versus bipolars. Proc Natl Acad Sci U S A. 2009;106(28):11731-11736.

28. Wang L, Lockstone HE, Guest PC, et al. Expression profiling of fibroblasts identifies cell cycle abnormalities in schizophrenia. J Proteome Res. 2010;9(1):521-527.

29. Okazaki S, Boku S, Otsuka I, et al. The cell cycle-related genes as biomarkers for schizophrenia. Progr Neuro psychopharmacol Biol Psychiatry. 2016;70:85-91.

30. Yurov YB, Iourov IY, Vorsanova SG, et al. The schizophrenia brain exhibits low-level aneuploidy involving chromosome 1. Schizophr Res. 2008;98(1-3):139-147.

31. Yurov YB, Vostrikov VM, Vorsanova SG, Monakhov VV, Iourov IY. Multicolor fluorescent in situ hybridization on post-mortem brain in schizophrenia as an approach for identification of low-level chromosomal aneuploidy in neuropsychiatric diseases. Brain Dev. 2001; 23(Suppl 1):S186-S190.

32. Kushima I, Aleksic B, Nakatochi M, et al. High-resolution copy number variation analysis of schizophrenia in Japan. Mol Psychiatry. 2017;22(3):430-440. 


\section{Supplementary materials}

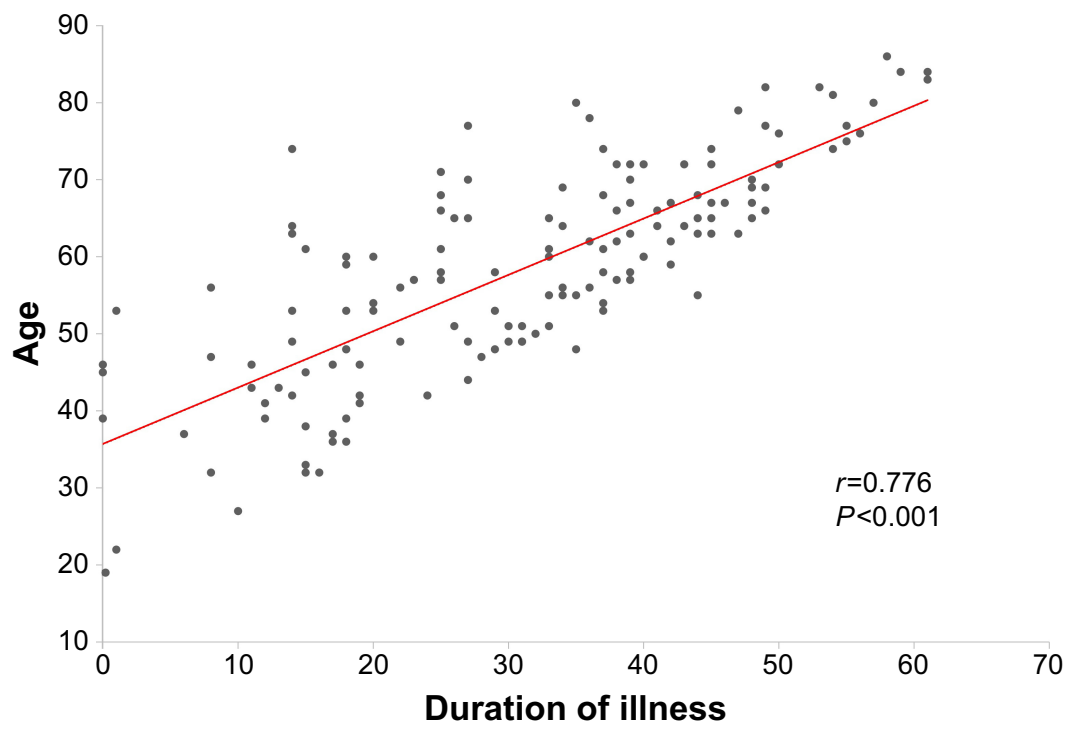

Figure SI Correlation between duration of illness and age in patients with schizophrenia. $P$-value and $r$ values were calculated using Spearman's rho test.

Table SI Comparisons of demographic and clinical characteristics between participants with and without LOY in the schizophrenia group

\begin{tabular}{llll}
\hline & $\begin{array}{l}\text { SCZ with LOY } \\
(\mathbf{n}=1 \mathbf{3})\end{array}$ & $\begin{array}{l}\text { SCZ without LOY } \\
(\mathbf{n = 8 0})\end{array}$ & P-value \\
\hline Age (years) (median [IQR]) & $68.0[66.0-76.0]$ & $65.0[60.0-72.0]$ & $0.077^{\mathrm{a}}$ \\
Duration of illness (years) (median [IQR]) & $49.0[43.0-53.0]$ & $38.0[27.0-44.3]$ & $0.0016^{\mathrm{a}}$ \\
Smoking (never/former/current) & $4 / 4 / 5$ & $18 / 28 / 34$ & $0.602^{\mathrm{b}}$ \\
Antipsychotic dose (mg/day) (median [IQR]) & $600.0[325.0-976.0]$ & $610.0[368.8-1,265.6]$ & $0.56 \mathrm{I}^{\mathrm{a}}$ \\
\hline
\end{tabular}

Notes: ${ }^{a}$-value calculated using the Mann-Whitney U-test. ${ }^{b} P$-value calculated using the Cochran-Armitage trend test. ${ }^{c}$ Antipsychotic dose was calculated based on chlorpromazine equivalents.

Abbreviations: LOY, loss of chromosome Y; SCZ, schizophrenia; IQR, interquartile range.

Table S2 Logistic regression analysis of LOY in peripheral blood samples of patients with schizophrenia

\begin{tabular}{lllll}
\hline Explanatory variable & \multicolumn{4}{l}{ Response variable: LOY $(\mathbf{Y} / \mathbf{X}$ ratio $<\mathbf{0 . 9})$} \\
\cline { 2 - 5 } & $\mathbf{B}^{\mathbf{a}}$ & $\mathbf{P}$-value & $\mathbf{O R}$ & $\mathbf{9 5 \%} \mathbf{C l}$ \\
\hline Age & 0.062 & 0.109 & 1.06 & $0.99-1.15$ \\
Smoking status & -0.109 & 0.775 & 0.90 & $0.42-1.89$ \\
Antipsychotic dose & -0.0001 & 0.745 & 1.00 & $0.99-1.00$ \\
\hline
\end{tabular}

Notes: For logistic regression analyses, LOY was the response variable, while age, smoking, and antipsychotic dose were the explanatory variables. ${ }^{\mathrm{B}} \mathrm{represents}$ the unstandardized partial regression coefficient.

Abbreviation: LOY, loss of chromosome Y.

Table S3 Smoking rates in Japanese men

\begin{tabular}{llllll}
\hline Age (years) & \multicolumn{3}{l}{ Smoking rate in Japan (\%) } & & \\
\cline { 2 - 6 } & $\mathbf{2 0 - 2 9}$ & $\mathbf{3 0 - 3 9}$ & $\mathbf{4 0 - 4 9}$ & $\mathbf{5 0 - 5 9}$ & $\mathbf{6 0 - 6 9}$ \\
\hline General population $^{\mathrm{a}}$ & 37.6 & 43.2 & 43.2 & 41.0 & 31.9 \\
Schizophrenia $^{b}$ & 0 & 33.3 & 50.0 & 45.2 & 38.1 \\
\hline
\end{tabular}

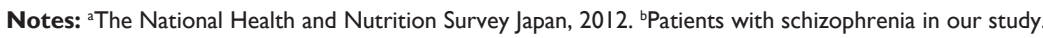




\section{Publish your work in this journal}

Neuropsychiatric Disease and Treatment is an international, peerreviewed journal of clinical therapeutics and pharmacology focusing on concise rapid reporting of clinical or pre-clinical studies on a range of neuropsychiatric and neurological disorders. This journal is indexed on PubMed Central, the 'PsycINFO' database and CAS,

and is the official journal of The International Neuropsychiatric Association (INA). The manuscript management system is completely online and includes a very quick and fair peer-review system, which is all easy to use. Visit http://www.dovepress.com/testimonials.php to read real quotes from published authors.

Submit your manuscript here: http://www.dovepress.com/neuropsychiatric-disease-and-treatment-journal 